\title{
Automatic text difficulty classifier: Assisting the selection of adequate reading materials for European Portuguese teaching
}

\author{
Pedro Curto ${ }^{1,2}$, Nuno Mamede ${ }^{1,2}$ and Jorge Baptista ${ }^{1,3}$ \\ ${ }^{1}$ Instituto Superior Técnico, Universidade de Lisboa, Av. Rovisco Pais, 1049-001 Lisboa, Portugal \\ 2 INESC-ID Lisboa/L2F - Spoken Language Lab, R. Alves Redol, 9, 1000-029 Lisboa, Portugal \\ ${ }^{3}$ Universidade do Algarve/FCHS and CECL, Campus de Gambelas, 8005-139 Faro, Portugal \\ pedro.curto,nuno.mamede@l2f.inesc-id.pt, jbaptis@ualg.pt
}

Keywords: Readability, Readability assessment metrics, Automatic readability classifier, Linguistic features extraction, Portuguese.

\begin{abstract}
This paper describes a system to assist the selection of adequate reading materials to support European Portuguese teaching, especially as second language, while highlighting the key challenges on the selection of linguistic features for text difficulty (readability) classification. The system uses existing Natural Language Processing (NLP) tools to extract linguistic features from texts, which are then used by an automatic readability classifier. Currently, 52 features are extracted: parts-of-speech (POS), syllables, words, chunks and phrases, averages and frequencies, and some extra features. A classifier was created using these features and a corpus, previously annotated by readability level, using a five-levels language classification official standard for Portuguese as Second Language. In a five-levels (from A1 to C1) scenario, the best-performing learning algorithm (LogitBoost) achieved an accuracy of $75.11 \%$ with a root mean square error (RMSE) of 0.269. In a three-levels (A, B and C) scenario, the best-performing learning algorithm (C4.5 grafted) achieved $81.44 \%$ accuracy with a RMSE of 0.346 .
\end{abstract}

\section{INTRODUCTION}

The selection of adequate reading materials for educational purposes is an important task for teaching languages, since giving students reading materials that are "too difficult" or "too easy" can both hinder the learning process and demotivate the students (Fulcher, 1997). This task implies measuring the text readability, or text difficulty, which remains today a relevant research topic, and in the case of Portuguese language teaching, it is still performed mostly manually.

This paper presents an automatic classifier for European Portuguese texts, based on a variety of linguistic features. It seeks to assist the selection of adequate reading materials for teaching European Portuguese, especially as a second language, adjusting them to different language proficiency levels. However, assigning readability scores to texts is also important in other areas, such as in the production of medical information, tools and software manuals, safety instruc- tions, etc., whose correct interpretation is essential to avoid different types of risk and to make such texts accessible reading to the majority of the population.

The extraction of linguistic features from texts is a core task in the creation of automatic readability classifiers. Text readability is affected, among other factors, both by lexical difficulty (the vocabulary level) and by the syntactic difficulty (the sentence complexity) (Klare, 1963). This paper presents a system that automatically extracts linguistic features from Portuguese texts and an automatic readability classifier for European Portuguese texts. To accomplish this, the system uses existing Natural Language Processing (NLP) tools, a parser and an hyphenator, and two corpora, previously annotated by readability level. Currently, the system extracts 52 features, grouped in 7 groups: parts-of-speech (POS), syllables, words, chunks and phrases, averages and frequencies, and some extra features.

Two experiments were carried out to evaluate the classification task: one based on a five-levels scale 
(A1, A2, B1, B2, C1), taken from the Framework for Teaching Portuguese Abroad (in Portuguese, Quadro de Referência para o Ensino de Português no Estrangeiro, QuaREPE) ${ }^{1}$, published by the Portuguese Ministry of Education and Science (Grosso et al., 2011a), and a second experiment based in a simplified three-levels scale (A, B and C).

The paper is organized as follows: first, some related work is presented (Section 2), and then the Natural Language Processing tools here used (Section 3), followed by the features extracted from the text (Section 4) and the automatic readability classifier here developed (Section 5). Finally, the evaluation (Section 6) is presented, followed by the conclusions and perspectives for future work (Section 7).

\section{RELATED WORK}

There are several works on the topic of feature extraction for predicting the readability of documents. For English, early approaches consisted only in measuring simple features like the average sentence length, average number of syllables per word, etc. These methods include metrics such as Flesch Reading Ease (Flesch, 1943), the Fog Index (Gunning, 1952; Gunning, 1969), the Fry Graph (Fry, 1968) and the SMOG ("Simple Measure of Gobbleygook") (McLaughlin, 1969). In general, these methods do not take into account the content of documents, which was only later considered for readability metrics, when some systems used a pre-determined list of words to predict the reading difficulty, such as the Lexile (Stenner, 1996) measure. More recently, language models have been used instead for this task, such as unigram language models, trained to predict the reading difficulty of English documents (Thompson and Callan, 2004). Other methods used syntactic features in addition to the language models (Schwarm and Ostendorf, 2005), while some approaches (Pitler and Nenkova, 2008) relied on a variety of linguistic features, namely lexical, syntactic and discourse relations, in order to improve the classification.

Regarding the systems developed for Portuguese that are able to assess the readability of texts based on linguistic features' extraction, one can refer REAP.PT ${ }^{2}$ (Marujo et al., 2009) ("REAder-specific Practice for Portuguese"), a tutoring system for Euro-

\footnotetext{
1 http://www.dgidc.min-edu.pt/ outrosprojetos/data/outrosprojectos/Portugues/ Documentos/manual_quarepe_orientador_versao_ final_janeiro_2012.pdf (accessed in Dec. 2014).

2 http://call.12f.inesc-id.pt/reap.public (accessed in Dec. 2014).
}

pean Portuguese vocabulary learning, which has been developed from the REAP system (Brown and Eskenazi, 2004) (English). Its readability measurement task is based on lexical features, such as statistics of word unigrams. It achieved an adjacent accuracy of $87.60 \%$ and an RMSE of 0.676 on 10-fold cross validation. LX-CEFR ${ }^{3}$ (Branco et al., 2014) is yet another system to select adequate materials for creating exams for teaching European Portuguese as second language. Its readability measurement task is based on the Flesch Reading Ease formula, frequency of nouns, average syllables per word, and average words per sentence. It achieved an maximum accuracy of $30 \%$ on 10 -fold cross validation, while only using the average number of syllables per word in the classification task.

\section{NATURAL LANGUAGE PROCESSING TOOLS}

To aid the extraction of features from European Portuguese texts, the system uses the natural language processing chain STRING $^{4}$ (Statistical and RuleBased Natural Language Processing chain) (Mamede et al., 2012) to extract statistical information about the texts. The number of syllables is extracted using the hyphenator YAH (Yet Another Hyphenator) (Figueirinha, 2013).

STRING (Mamede et al., 2012) is an hybrid statistical and rule-based natural language processing chain for Portuguese, which has been developed by L2FSpoken Language Laboratory, at INESC-ID Lisboa. STRING has a modular structure and performs all the basic NLP tasks, namely tokenization and text segmentation, part-of-speech tagging, rule-based and statistical morphosyntactic disambiguation, shallow parsing (chunking) and deep parsing (dependency extraction). For parsing, the system uses $X I P^{5}$ (AitMokhtar et al., 2002) (Xerox Incremental Parser), a rule-based parser, whose European Portuguese grammar was jointly developed with XEROX.

The YAH Hyphenator (Figueirinha, 2013) is a tool that has been developed by L2F-Spoken Language Laboratory, at INESC-ID Lisboa, originally designed by Ricardo Ribeiro and later improved by Pe-

\footnotetext{
3 http://nlx.di.fc.ul.pt/ jrodrigues/camoes/ indexLXCENTER. html? exemplo (accessed in Dec. 2014).

4 https://string.12f.inesc-id.pt (accessed in Dec. 2014).

${ }^{5}$ Reference Guide: https: //open.xerox.com/Repo/ service/XIPParser/public/XIPReferenceGuide.pdf (accessed in Dec. 2014).
} 
dro Figueirinha. This is a rule-based system that applies various word processing division rules.

\section{FEATURES}

The set of 52 features extracted by the system consists in: (i) part-of-speech (POS) tags, chunks, words and sentences features; (ii) verb features and different metrics involving averages and frequencies; (iii) several metrics involving syllables; and (iv) extra features. The features of group (i) are extracted from the chunking tree generated by STRING; features from groups (ii) and (iv) are also extracted from the chunking tree, but complemented by the dependencies' information generated by the processing chain; the metrics related to syllables (iii) are extracted using YAH. The feature set used is present in appendix section.

For lack of space, only a sketch of the rationale behind these features is provided below; see (Curto, 2014) for details.

The system calculates the part-of-speech (POS) relative percentages. Conceptual information, often introduced through nouns and named entities, e.g. people's names, locations, organizations, etc., is important in text comprehension, yet the more entities and types of entities a text has, the harder it is to keep track of them and of the relations between them.

Statistics about elementary syntactic constituents (or chunks: nominal phrases - NP, prepositional phrases - PP, etc.) are also extracted. Auxiliary verb chunks (Baptista et al., 2010) can combine among them to form longer, complex verbal chains: the longer the chain, the more complex is the decoding of the grammatical values involved. Subclause chunks are related to sentence hypotaxis complexity (Beaman, 1984), while the number of coordination relations and the length of their chains are related with the parataxis complexity.

The length of a text is related with its readability, i.e. typically, longer texts, specially with long sentences, have much more detail or content, which can make them more difficult to understand. Word frequency is related to the vocabulary use and, according to (Thompson and Callan, 2004), it can affect the readability of a text: texts with more familiar vocabulary are easier to understand by the reader. Word frequency has been captured by way of a unigram-based language model, defined by:

$$
\sum_{w} C(w) \times \log (P(w \mid M))
$$

where $P(w \mid M)$ is the probability of word $w$ according to a background corpus $M$, and $C(w)$ is the number of times $w$ appears in the text. This model will be biased in favor of shorter texts. Since each word has probability less than 1 , the $\log$-probability of each word is less than 0 , and hence including additional words decreases the log-likelihood. To overcome this issue, the system calculates this probability in $\mathrm{n}$ groups of 50 words each and then calculates an average of the $n$ results. The calculations were performed using Laplace smoothing over the word frequencies, obtained from a set of several, distinct European Portuguese corpora, provided by the AC/DC project and available at Linguateca.

Based on previous statistics, the system then extracts several averages and frequencies. The frequency of nouns is the ratio of the number of nouns per number of words, and a similar ratio is calculated for the verbs. The average number of verb phrases per sentence and the average length of sentences derive from Pitler and Nenkova (Pitler and Nenkova, 2008): the more verbs a sentence contains and the longer a sentence is, the more complicated it becomes to understand it. The average length of syllables per word is deemed important for readability metrics such as the Flesch Reading Ease and others metrics (see §2).

The number of pronouns per noun phrases derives from CohMetrixPort system (Scarton and Aluísio, 2010). The greater the number of pronouns per noun phrases, the more difficult it becomes to identify who or what the pronoun refers to. The use of NP with a definite or demonstrative determiner usually implies a process of reference resolution, as opposed to indefinite determiners, which do not refer to previously occurring words. A text with lower definite/indefinite NP ratio should be more cohesive, hence the anaphora processing involved renders its decoding more difficult.

The feature extraction system was evaluated on a manually annotated text, with 490 words and 14 sentences taken from journalistic texts. For lack of space detailed analysis can not be made here. The system achieve $98.81 \%$ of precision, $98.88 \%$ recall and a Fmeasure (F) of $98.85 \%$.

The feature set is largely language-independent, though some features require adequate NLP tools (e.g. chunking), while others depend on the morphosyntactic properties of the language (e.g. auxiliary verb types). Specific language-dependent features, to be explored in future work, relate mostly to syntactic dependencies (e.g. modifier, adjunct), but they can be approximated using broad interpretation of those relations. 
Table 1: Corpus distribution.

\begin{tabular}{lrrrrrrrrrrr}
\hline & A1 & \% & A2 & \% & B1 & \% & B2 & \% & C1 & \% & Total \\
\hline \# Text & 29 & 12.2 & 39 & 16.5 & 136 & 57.4 & 14 & 5.9 & 19 & 8.0 & 237 \\
\# Sentences & 184 & 11.9 & 384 & 24.7 & 535 & 34.5 & 199 & 12.8 & 250 & 16.1 & 1552 \\
\# Words & 2,655 & 10.3 & 5,010 & 19.4 & 9,407 & 36.3 & 3,702 & 14.2 & 5,114 & 19.8 & 25,888 \\
\hline
\end{tabular}

\section{READABILITY CLASSIFIER}

According to the Framework for Teaching Portuguese Abroad (in Portuguese, Quadro de Referência para o Ensino de Português no Estrangeiro, QuaREPE) (Grosso et al., 2011a), published by the Ministry of Education and Science, and based on the international standards of the European Common Reference Framework for Languages, it is considered that the degree of proficiency in a foreign language can be determined on a scale of five-levels: A1: initiation; A2: elementary; B1: intermediate; B2: upper intermediate; and $\mathrm{C} 1$ : advanced.

The system's performance on the classification task was evaluated with two experiments: one based on this five-levels scale and a second experiment based on a simplified three-levels scale, i.e., the classifier is trained to predict if the text belongs to level $\mathrm{A}, \mathrm{B}$ or $\mathrm{C}$. This second experiment is useful because distinguishing between the levels A1 and A2; B1 and B2 may be very difficult, even for a specialist.

The corpus used to train the classifier consists of a set of 237 texts, provided by the Instituto Camões ${ }^{6}$ and previously classified according to their readability. This corpus was created from tests, exams and materials used for teaching European Portuguese. The manual text readability classification takes into account reading and comprehension skills stipulated by the QuaREPE for each level. Table 1 shows the corpus distribution for each readability level. One should bear in mind that the uneven distribution and the small size of some classes (and of the corpus as a whole), are likely to have an impact on the classifier, which is unavoidable due to the scarcity of resources for this task.

\section{EVALUATION}

\subsection{Readability Classifier}

In both scenarios, several machine learning algorithms available in WEKA machine learning

\footnotetext{
6 http://www.instituto-camoes.pt (accessed in Dec. 2014)
}

toolkit $^{7}$ (Bouckaert et al., 2013) were tested (Table 2 and 5). The evaluation was performed using 10 -fold cross-validation. The metrics chosen for measuring the performance of the classifier were accuracy (percentage of correctly classified instances), root mean square error (RMSE), ROC area and Kappa statistics. Additionally, a confusion matrix and algorithm performance comparison are presented for each scenario.

\subsubsection{Five-Levels Classification}

The best-performing learning algorithm was the $\log$ itBoost (Table 2).

Table 2: Algorithms comparison results (five-levels classifier)

\begin{tabular}{lcc}
\hline Algorithms & Accuracy & RMSE \\
\hline Naive Bayes & $68.35 \%$ & 0.339 \\
Support Vector Machines & $70.04 \%$ & 0.342 \\
Logistic regression & $59.07 \%$ & 0.402 \\
K-nearest neighbors learner & $65.40 \%$ & 0.368 \\
K* & $70.04 \%$ & 0.339 \\
AdaBoost & $59.49 \%$ & 0.360 \\
LogitBoost & $\mathbf{7 5 . 1 1 \%}$ & $\mathbf{0 . 2 6 9}$ \\
Holte's OneR & $69.20 \%$ & 0.351 \\
C4.5 & $71.31 \%$ & 0.323 \\
C4.5 grafted & $72.57 \%$ & 0.319 \\
Decision stumps & $61.18 \%$ & 0.297 \\
Random Forest & $70.04 \%$ & 0.275 \\
\hline
\end{tabular}

In this scenario, we also considered the adjacent accuracy within 1 grade level as a useful evaluation metrics. This is the percentage of predictions that are equal to or show one level of difference to the manually assigned level. Measuring strict accuracy is considered too demanding because manually assigned labels are not always consistent.

Table 3: Evaluation of the readability classifier (five-levels).

Accuracy RMSE ROC Area Kappa Adjacent Acc.

\begin{tabular}{lllll}
\hline $75.11 \%$ & 0.269 & 0.918 & 0.590 & $91.98 \%$
\end{tabular}

In this scenario (Table 3), the classifier correctly classified $75.11 \%$ instances, e.g., 178 texts. It is in-

\footnotetext{
7 http://www.cs.waikato.ac.nz/ml/ weka (accessed in Dec. 2014).
} 
teresting to notice that for most texts, the assigned level is either correct or mostly within one-level difference (Table 4). As expected, the adjacent accuracy is very high (91.98\%) and the RMSE result is low because the expected and the observed values are close. The Kappa metric is a chance-corrected measure of agreement between the classifications and the expected values, where 1.0 represents perfect agreement. The Kappa value obtained (0.59) corresponds to a moderate agreement, according to (Landis and Koch, 1977).

Table 4: Confusion Matrix (five-levels).

\begin{tabular}{ccccccc}
\hline & & \multicolumn{5}{c}{ Predicted class } \\
& & A1 & A2 & B1 & B2 & C1 \\
\hline \multirow{2}{*}{} & A1 & 18 & 7 & 4 & 0 & 0 \\
$\frac{\Xi}{0}$ & A2 & 2 & 27 & 10 & 0 & 0 \\
$\frac{\pi}{\Xi}$ & B1 & 5 & 4 & 121 & 1 & 5 \\
는 & B2 & 0 & 0 & 4 & 2 & 8 \\
C1 & 0 & 1 & 4 & 4 & 10 \\
\hline
\end{tabular}

\subsubsection{Three-Levels Classification}

In this scenario, the best-performing learning algorithm was the $C 4.5$ grafed (Table 5), with a $81.44 \%$ accuracy and 0.346 RMSE. The second best algorithm, and with very similar results, was the LogitBoost, which achieved a lower accuracy than the $C 4.5$ grafed $(80.17 \%)$ despite having a lower RMSE value (0.294). Since, in this scenario, the scale used has only 3 levels, the RMSE value was considered less significant than the accuracy value.

Table 5: Algorithms comparison results (three-levels classifier)

\begin{tabular}{lcc}
\hline Algorithms & Accuracy & RMSE \\
\hline Naive Bayes & $75.11 \%$ & 0.405 \\
Support Vector Machines & $75.11 \%$ & 0.363 \\
Logistic regression & $70.46 \%$ & 0.439 \\
K-nearest neighbors learner & $72.15 \%$ & 0.428 \\
K* & $77.22 \%$ & 0.385 \\
AdaBoost & $68.78 \%$ & 0.352 \\
LogitBoost & $80.17 \%$ & 0.294 \\
Holte's OneR & $73.84 \%$ & 0.418 \\
C4.5 & $80.17 \%$ & 0.352 \\
C4.5 grafted & $\mathbf{8 1 . 4 4 \%}$ & $\mathbf{0 . 3 4 6}$ \\
Decision stumps & $70.89 \%$ & 0.347 \\
Random Forest & $79.75 \%$ & 0.295 \\
\hline
\end{tabular}

The three-levels classification (Table 6) achieved a better accuracy $(86.32 \%)$ and obtained RMSE and ROC area values similar to the previously mentioned
Table 6: Evaluation of the readability classifier (threelevels).

\begin{tabular}{cccc}
\hline Accuracy & RMSE & ROC Area & Kappa \\
\hline $81.44 \%$ & 0.346 & 0.831 & 0.639 \\
\hline
\end{tabular}

classifier. In this scenario, the adjacent accuracy is not calculated. However, it is important to report that for all the texts corresponding to $\mathrm{A}$ or $\mathrm{C}$ levels, the level assigned is correct or within one-level difference (Table 7). The Kappa value obtained (0.639) corresponds to a substantial agreement, according to (Landis and Koch, 1977).

\begin{tabular}{|c|c|c|c|c|c|}
\hline & & & \multicolumn{3}{|c|}{$\begin{array}{l}\text { Predicted } \\
\text { class }\end{array}$} \\
\hline & & & A & B & $\mathrm{C}$ \\
\hline \multirow{3}{*}{ 丞 } & \multirow{3}{*}{$\frac{\tilde{y}}{0}$} & A & 57 & 11 & 0 \\
\hline & & B & 12 & 127 & 11 \\
\hline & & $\mathrm{C}$ & 0 & 10 & 9 \\
\hline
\end{tabular}

\subsection{Feature Contribution}

To assess the contribution of the features extracted for the readability classification, we used the WEKA toolkit (Bouckaert et al., 2013) with the feature selection algorithm InfoGainAttributeEval ${ }^{8}$. This evaluation was conducted in the two different, previously mentioned scenarios (Section 5). Figures 1 and 2 show the results for the features with higher contribution on the classification task.

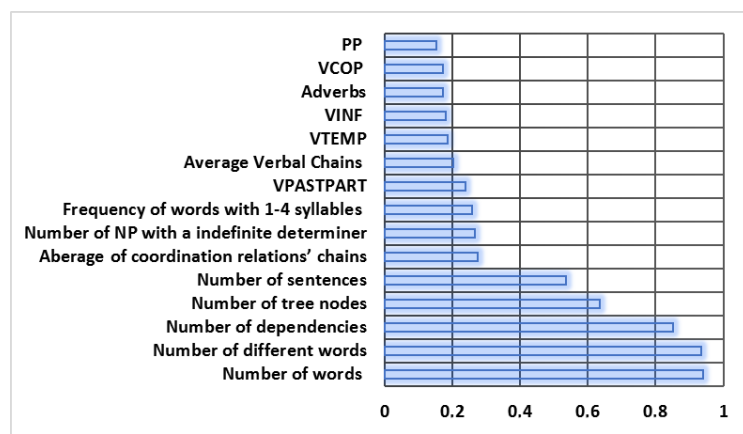

Figure 1: Feature contribution for the five-levels scale classification

Regarding the five-levels classification (Figure 1), among the top five features, some are computationally simple to obtain, namely the number of words

\footnotetext{
8 http://weka.sourceforge.net/doc.stable/ weka/attributeSelection/InfoGainAttributeEval. html (accessed in Dec. 2014).
} 
(0.94), of different words (0.93), and sentences (0.54), showing the relevance of more traditional readability metrics. On the other hand, the number of dependencies $(0.85)$ and the total number of nodes (0.64) result from the processing chain and justify the use of more sophisticated, NLP-based tools in this classification task. The remaining parameters are related to the POS groups (frequency of adverbs), phrases (frequencies of past participle verb phrases - VPASTPART, temporal auxiliary verb phrases - VTEMP, infinitive verb phrases - VINF, copulative verb phrases - VCOP and $\mathrm{PP}$ ) and average and frequencies (average of coordinating relations' chains, frequency of words with 1-4 syllables and average of verbal chains) and extra features (number of noun phrases - NP - with indefinite determiners).

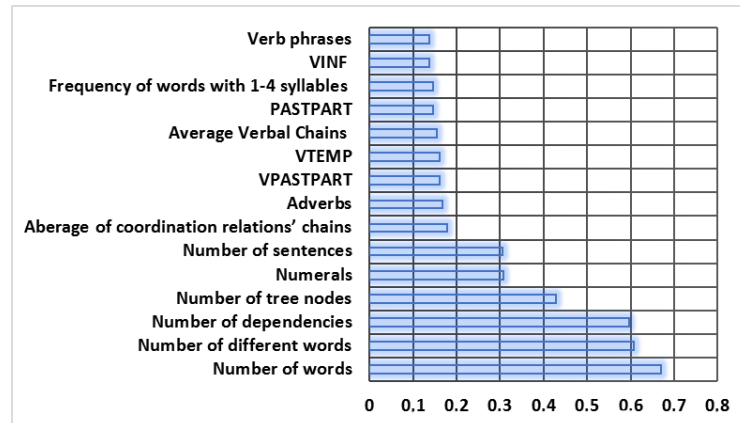

Figure 2: Feature contribution for the three-levels scale classification

In the three-levels classification (Figure 2), the features that contributed most to the success of the classifier were: the number of words (0.67), of different words (0.61) and of dependencies (0.60). Again, this highlights the importance of using a more sophisticated NLP-based tool and simple counts of words and sentences length for the classification task. The list of fifteen features that stood out in the success of the two classifiers is very similar (Spearman correlation coefficient of 0.881 ), only changing the priority order of some features. The 1-4 syllables word frequency parameter was not so relevant to the results of the tree-levels scenario $(0.14)$ as it was in the fivelevels scenario (0.26).

Additionally, box plot diagrams were built for each feature used, which allowed to analyse feature value variations between the different readability levels. Given the large number of diagrams, only two examples are presented here, namely, the one referring to the number of different words (Figure 3) and another on the average of verbal chain's (Figure 4). By analysing the box plot diagrams, it was possible to conclude that there is no feature that has completely different values for each readability level and, on the features with highest contribution such as the one on Figure 3 (0.93 in the five-levels and 0.61 on the threelevels scale classification), we observe that the B1 level has texts that seems to belong to A1 and A2 levels. However, the remaining features (with lowest contribution), like the average size of the verbal chain (Figure 4), do not have a distinct values range between different readability levels. These observations confirm the complexity of the text readability classification task.

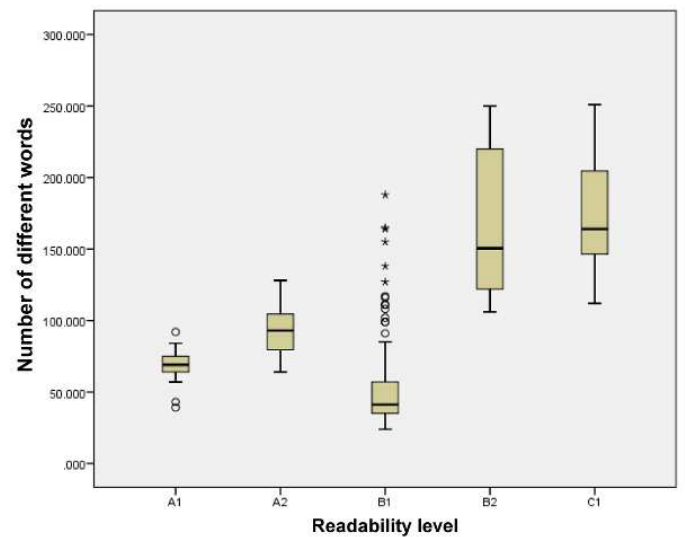

Figure 3: Number of different words value variations between the different readability levels

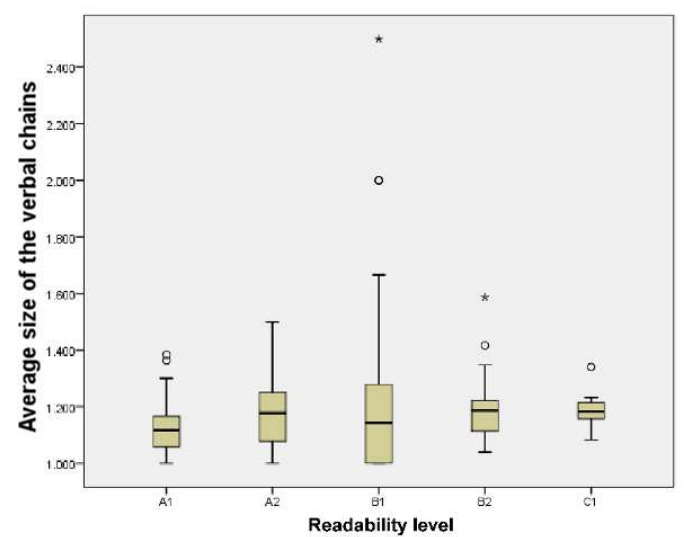

Figure 4: Average size of the verbal chain value variations between the different readability levels

\section{CONCLUSIONS AND FUTURE WORK}

This paper presented two classifiers for European Portuguese texts based on a variety of linguistic features. These classifiers seek to assist the selection of adequate reading materials for teaching European Por- 
tuguese as a second language adapted to different language proficiency levels.

The feature system focused on 52 features, from simple word counts to complex syllables and wordlength counts, and rather sophisticated data involving parsing techniques. The feature extraction achieved $98.85 \% \mathrm{~F}$-measure, which is quite satisfactory.

A study of the features that contributed most to the success of the classification task was conducted. For both classifiers, the feature contribution shows the importance of using more sophisticated, NLP-based tools in this classification task. Additionally, the complexity of the classification task was shown by the analysis of the feature value variations between the different readability levels.

In both scenarios, with five readability levels (A1 to $\mathrm{C} 1$ ) or with three-levels (A, B or C), the classifiers here developed achieved good results with an accuracy of $75.11 \%$ and $81.44 \%$, respectively, and most of their errors are within one-level distance from the expected results. For comparative proposes, the five-levels classifier developed presents good results against the best classifier of the LX-CEFR system (Branco et al., 2014)(section 2), which just got a maximum accuracy of $30 \%$, while only using the average number of syllables per word in the classification task. For evaluation proposes, the corpus used in the classifiers here presented is the same used by LX-CEFR system but with more 112 texts.

In the future, the system here presented will be made available to the general public through a web form and it can easily be extended by adding new features or metrics of interest to the task at hand. Taking into account the small size of the corpus annotated according to the readability level in the five-level scale defined by QuaREPE (Grosso et al., 2011b), it may prove useful to investigate unsupervised learning techniques, i.e. techniques that do not depend on a previously classified corpus. For example, using techniques of cluster analysis, which allows to group a set of objects into clusters via their similarities.

\section{ACKNOWLEDGEMENTS}

This work was supported by national funds through Fundação para a Ciência e a Tecnologia (FCT) with reference UID/CEC/50021/2013. The authors gratefully acknowledge the use of the corpus classified according to the Framework for Teaching Portuguese Abroad and provided by the Instituto Camões.

\section{REFERENCES}

Aït-Mokhtar, S., Chanod, J.-P., and Roux, C. (2002). Robustness Beyond Shallowness: Incremental Deep Parsing. Natural Language Engineering, 8(3):121144.

Baptista, J., Mamede, N., and Gomes, F. (2010). Auxiliary Verbs and Verbal Chains in European Portuguese. In Proceedings of the $9^{\text {th }}$ International Conference on Computational Processing of the Portuguese Language (PROPOR'10), pages 110-119, Porto Alegre, RS, Brazil. Springer.

Beaman, K. (1984). Coordination and Subordination Revisited: Syntactic Complexity in Spoken and Written Narrative Discourse. In Coherence in Spoken and Written Discourse, volume 12, pages 45-80. Ablex, Norwood, NJ.

Bouckaert, R. R., Frank, E., Hall, M., Kirkby, R., Reutemann, P., Seewald, A., and Scuse, D. (2013). WEKA Manual for Version 3-7-11. Hamilton, New Zealand.

Branco, A., Rodrigues, J., Costa, F., Silva, J., and Vaz, R. (2014). Rolling out Text Categorization for Language Learning Assessment Supported by Language Technology. In Proceedings of the $11^{\text {th }}$ International Conference on Computational Processing of Portuguese (PROPOR'14), volume 8775, pages 256-261, São Carlos, Brazil.

Brown, J. and Eskenazi, M. (2004). Retrieval of Authentic Documents for Reader-Specific Lexical Practice. In Proceedings of InSTIL/ICALL Symposium 2004, volume 17, pages 25-28, Venice, Italy.

Curto, P. (2014). Classificador de textos para o ensino de português como segunda língua. Master's thesis, Instituto Superior Técnico - Universidade de Lisboa, Lisboa.

Figueirinha, P. (2013). Syntactic REAP.PT. Exercises on Word Formation. Master's thesis, Instituto Superior Técnico - Universidade de Lisboa, Lisboa.

Flesch, R. (1943). Marks of Readable Style: A Study in Adult Education (Contributions to education). Number 897. Columbia University, Teachers College, Bureau of Publications, New York, United States.

Fry, E. (1968). A readability formula that saves time. Journal of Reading, 11(7):513-578.

Fulcher, G. (1997). Text difficulty and accessibility: Reading formulae and expert judgement. System, 25(4):497-513.

Grosso, M. J., Soares, A., de Sousa, F., and Pascoal, J. (2011a). QuaREPE - Quadro de Referência para o Ensino de Português no Estrangeiro. Lisboa: MEC/DGIDC.

Grosso, M. J., Soares, A., de Sousa, F., and Pascoal, J. (2011b). QuaREPE - Quadro de Referência para o Ensino de Português no Estrangeiro. Documento Orientador. Lisboa: Ministério da Educação e Ciência/Direção Geral de Inovação e Desenvolvimento Curricular, http://www.dgidc.min-edu. pt/outrosprojetos/data/outrosprojectos/ Portugues/Documentos/manual_quarepe_ orientador_versao_final_janeiro_2012.pdf. 
Gunning, R. (1952). The Technique of Clear Writing. McGraw-Hill, New York, USA.

Gunning, R. (1969). The FOG Index after twenty years. Journal of Business Communication, 6(2):3-13.

Klare, G. (1963). The measurement of readability. Iowa State University Press, Ames, USA.

Landis, J. R. and Koch, G. G. (1977). The measurement of observer agreement for categorical data. Biometrics, pages 159-174.

Mamede, N., Baptista, J., Diniz, C., and Cabarrão, V. (2012). STRING: An Hybrid, Statistical and RuleBased Natural Language Processing Chain for Portuguese. In Proceedings of the $10^{\text {th }}$ International Conference on Computational Processing of Portuguese (PROPOR'12), volume Demo Session, Coimbra, Portugal, https://string.12f.inesc-id.pt/ w/index.php/Publications.

Marujo, L., Lopes, J., Mamede, N., Trancoso, I., Pino, J., Eskenazi, M., Baptista, J., and Viana, C. (2009). Porting REAP to European Portuguese. In Proceedings of SLaTE 2009, pages 69-72, Wroxall Abbey Estate, Warwickshire, England.

McLaughlin, G. H. (1969). SMOG grading: A new readability formula. Journal of Reading, 12(8):639-646.

Pitler, E. and Nenkova, A. (2008). Revisiting readability: a unified framework for predicting text quality. In Proceedings of EMNLP'08, pages 186-195, Stroudsburg, PA, USA. ACL.

Scarton, C. E. and Aluísio, S. M. (2010). Análise da inteligibilidade de textos via ferramentas de Processamento de Língua Natural: adaptando as métricas do Coh-Metrix para o Português. Linguamática, 2(1):4561.

Schwarm, S. E. and Ostendorf, M. (2005). Reading level assessment using support vector machines and statistical language models. In Proceedings of ACL'05, pages 523-530, Stroudsburg, PA, USA. ACL.

Stenner, A. J. (1996). Measuring reading comprehension with the Lexile framework. In Fourth North American Conference on Adolescent/Adult, London, UK. Academic Press Ltd.

Thompson, K. C. and Callan, J. P. (2004). A Language Modeling Approach to Predicting Reading Difficulty. In Proceedings of NAACL'04, pages 193-200, Boston, United States. ACL.

\section{APPENDIX}

The list of features used in the classification task are presented in Table 8. For the classification task, the features' values for the parts-of-speech, chunks, verbs and extras groups (with the exception of total number of dependencies, total number of tree nodes and number of pronouns per NP) are represented by a ratio weighted by number of words divided by 1000 . For example, the adjectives' feature is calculated as follows: number of adjectives/(number of words/1000).
For the verbs group of features, the system considers the different inflected verbs forms as independent counts to measure the use of different tenses and verb forms. The special symbols are, for example, "\$”, "\%", “\#”, etc. 
Table 8: Features used in classification

\begin{tabular}{|c|c|}
\hline Group & Features \\
\hline Part-of-speech (POS) & $\begin{array}{l}\text { Adjectives (ADJ) } \\
\text { Adverbs (ADV) } \\
\text { Articles (ART) } \\
\text { Conjunctions (CONJ) } \\
\text { Interjections (INTERJ) } \\
\text { Nouns (NOUN) } \\
\text { Numerals (NUM) } \\
\text { Past participles (PASTPART) } \\
\text { Prepositions (PREP) } \\
\text { Pronouns (PRON) } \\
\text { Punctuation (PUNCT) } \\
\text { Special symbols (SYMBOL) }\end{array}$ \\
\hline Chunks & $\begin{array}{l}\text { Nominal phrases (NP) } \\
\text { Adjectival phrases (AP) } \\
\text { Prepositional phrases (PP) } \\
\text { Adverbial phrases (ADVP) } \\
\text { Temporal auxiliary verb phrases (VTEMP) } \\
\text { Aspectual auxiliary verb phrases (VASP) } \\
\text { Modal auxiliary verb phrases (VMOD) } \\
\text { Copulative verb phrases (VCOP) } \\
\text { Past participle verb phrases (VPASTPART) } \\
\text { Gerundive verb phrases (VGER) } \\
\text { Infinitive verb phrases (VINF) } \\
\text { Finite verb phrases (VF) } \\
\text { Sub-clause phrases (SC e REL) } \\
\text { Verb phrases (VF e VCOP) }\end{array}$ \\
\hline Sentences and words & $\begin{array}{l}\text { Number of sentences } \\
\text { Number of words } \\
\text { Number of different words } \\
\text { Words frequencies }\end{array}$ \\
\hline Verbs & $\begin{array}{l}\text { Number of different verbs forms } \\
\text { Number of auxiliary verbs } \\
\text { Number of main verbs }\end{array}$ \\
\hline Averages and frequencies & $\begin{array}{l}\text { Average number of verb phrases per sentence } \\
\text { Average length of sentences } \\
\text { Average length of syllables per word } \\
\text { Average size of verbal chains } \\
\text { Average size of coordination relation's chains } \\
\text { Frequency of verbs } \\
\text { Frequency of words with } 1-4 \text { syllables } \\
\text { Frequency of words with more than } 4 \text { syllables }\end{array}$ \\
\hline Extras & $\begin{array}{l}\text { Total number of dependencies } \\
\text { Total number of tree nodes } \\
\text { Number of pronouns per noun phrases (NP) } \\
\text { Number of NP with a definite or demonstrative determiner } \\
\text { Number of NP with a indefinite determiner } \\
\text { Number of subordinate clauses (SC/REL chunks) } \\
\text { Number of coordination relations } \\
\text { Number of omit subjects } \\
\text { Flesch Reading Ease BR readability measure }\end{array}$ \\
\hline
\end{tabular}

